\title{
Indicaciones inhabituales de trasplante hepático. Resultados
}

\author{
Andrés Troncoso T. ${ }^{1}$, Eduardo Briceño V. ${ }^{1}$, Alejandra Cancino M. ${ }^{2}$, Paulina Dellepiane M. ${ }^{2}$, \\ Humberto Soriano B. ${ }^{3}$, Juan Gana A. ${ }^{3}$, Carlos Benítez G. ${ }^{3}$, Rodrigo Wolff R. ${ }^{4}$, \\ Francisco Barrera M. ${ }^{4}$, Rodrigo Montaña R. ${ }^{5}$, Mario Concha P. ${ }^{5}$, Juan Carlos Pattilo S. ${ }^{6}$, \\ Nicolás Jarufe C. ${ }^{1}$, Juan Francisco Guerra C. ${ }^{1}$, Martin Dib M. ${ }^{1}$ y Jorge Martínez C. ${ }^{1}$
}

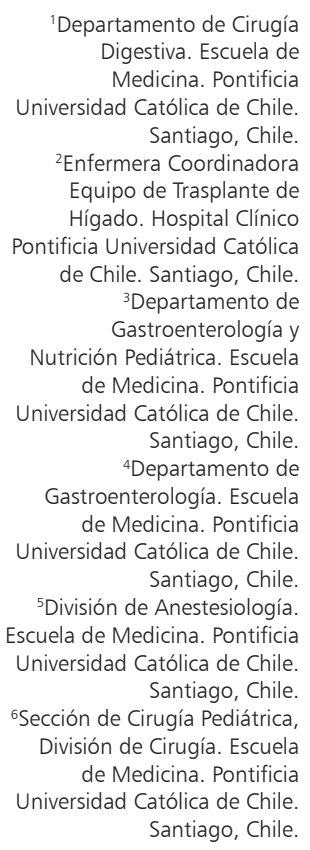

Recibido el 14 de octubre

de 2019 y aceptado para publicación el 6 de marzo de

Correspondencia a: Dr. Jorge Martínez C. jjmartinezc@gmail.com

\section{Unusual indications for liver transplantation. Results}

Introduction: Liver transplantation (LT) is an established therapy in the treatment of several acute and chronic end-stage liver diseases and hepatocellular carcinoma (HCC). The main indications worldwide are cirrhosis of different etiologies, HCC, biliary atresia in children, and fulminant hepatic failure (FHF). Less than $10 \%$ concerns unusual indications which include patients with miscellaneous diseases among which are hepatic polycystic disease (HPD), metabolic diseases (Niemann-Pick, others), portal/hepatopulmonary syndrome, metastasis of different tumors, among others. Aim: The objective of the study is to describe and asses the results obtained with liver transplantation in these indications. Materials and Method: We performed a non-concurrent cohort study that included all LT due to unusual indications between March 1997 and December 2016 in a university medical center. Of 295 TH performed, 34 (11.75\%) were due to these indications. Results: The most frequent causes were the portal/hepatopulmonary syndrome in 11 $(40.7 \%)$ patients and HPD in $9(26.5 \%)$. Metabolic diseases accounted for the third indication in $5(14.7 \%)$ cases. Seven (20.6\%) patients were less than 18 years old. The most frequent complications were biliary and hepatic artery thrombosis (HAT) in $6(17.6 \%)$ and $4(11.8 \%)$ cases, respectively. Patients complicated by a HAT suffered a massive EPH. Four $(12.5 \%)$, required retransplantation. Mortality at 90 days was 2 (5.9\%). Conclusion: LT is a feasible option in this group of patients with results similar to those obtained in classic indications of LT.

Key words: transplant; cirrhosis; hepatectomy.

\section{Resumen}

Introducción: El trasplante hepático (TH), es una terapia establecida en el tratamiento de diversas enfermedades del hígado agudas y crónicas terminales y del carcinoma hepatocelular (CHC). Las principales indicaciones en nuestro medio son la cirrosis de diferentes etiologías, el CHC, la atresia de vías biliares en niños y la falla hepática fulminante (FHF). Menos del 10\% corresponden a indicaciones inhabituales, que incluyen pacientes con una miscelánea de enfermedades entre las cuales están la enfermedad poliquística hepática (EPH), enfermedades metabólicas (Niemann-Pick, otras), el síndrome hepato/portopulmonar, metástasis de diferentes tumores, etc. Objetivo: Describir y evaluar los resultados obtenidos con el trasplante hepático en estas indicaciones. Materiales y Método: Estudio de cohorte no concurrente que incluyó los TH por indicaciones inhabituales realizados entre marzo de 1997 y diciembre de 2016. De 295 TH realizados, 34 (11,5\%) fueron por estas indicaciones. Resultados: Las causas más frecuentes fueron el síndrome porto/hepatopulmonar en $11(40,7 \%)$ pacientes y la EPH en $9(26,5 \%)$. Las enfermedades metabólicas representaron la tercera indicación, con $5(14,7 \%)$ casos. Siete $(20,6 \%)$ pacientes eran menores de 18 años. Las complicaciones más frecuentes fueron biliares y la trombosis de arteria hepática en $6(17,6 \%)$ y $4(11,8 \%)$ casos respectivamente; estos últimos eran portadores de una EPH masiva. Cuatro $(12,5 \%)$ pacientes requirieron retrasplante. La mortalidad a 90 días fue de $2(5,9 \%)$ enfermos. Conclusión: El TH es una opción factible en este grupo de pacientes con resultados similares a los obtenidos en las indicaciones clásicas.

Palabras clave: trasplante; cirrosis; hepatectomía. 


\section{Introducción}

El trasplante hepático (TH) es, actualmente, el mejor tratamiento de la insuficiencia hepática aguda y crónica terminal y de algunos cánceres del hígado'. Las principales indicaciones del TH corresponden a cirrosis hepática secundaria a virus de hepatitis B (VHB) o C (VHC), alcohol (OH), esteatohepatitis no alcohólica (NASH: por su abreviatura en inglés non-alcoholic steatohepatitis) y enfermedades colestásicas (cirrosis biliar primaria, colangitis esclerosante primaria). Menos del 10\% de los trasplantes son por indicaciones inhabituales ${ }^{2}$. Dentro de éstas, podemos encontrar enfermedades metabólicas (enfermedad de Niemann-Pick), vasculares (Rendu-Osler-Weber o telangiectasia hemorrágica hereditaria), tumores no hepatocelulares (hemangioendotelioma epitelial, tumores neuroendocrinos), congénitas (enfermedad poliquística) y por trauma. En varias de estas indicaciones los pacientes no tienen insuficiencia hepática ni hipertensión portal, por lo que el trasplante se realiza para mejorar la calidad de vida y/o sobrevida. Está descrito que el score de MELD (Model for End-Stage Liver Disease), no otorga la correcta prioridad a estos pacientes, por lo que deben ser seleccionados por un comité de expertos ${ }^{1}$.

El objetivo de este estudio es describir y evaluar los resultados obtenidos en nuestro centro con el trasplante hepático en estas indicaciones.

\section{Material y Método}

Se realizó un estudio de cohorte no concurrente que incluyó todos los TH realizados en nuestro centro por indicaciones inhabituales entre marzo de 1997 y diciembre de 2016. Se utilizó la base de datos de registro prospectivo, especialmente diseñada para casos de TH, que incluyó las variables biodemográficas, la indicación del trasplante, la necesidad de retrasplante, la morbilidad (complicaciones vasculares, biliares e infecciosas) y la mortalidad. La morbimortalidad fue analizada para este trabajo hasta los 90 días, y se realizó un análisis diferenciado para los pacientes pediátricos $(<18$ años). Se seleccionó todas aquellas indicaciones de TH inhabituales ${ }^{2}$ y se comparó los resultados clínicos obtenidos en ellos, con los resultados de aquellos trasplantes realizados por indicaciones clásicas.

Aspectos de la técnica quirúrgica y de los esquemas de tratamiento inmunosupresor han sido publicados previamente ${ }^{1,3}$ y no difieren mayormente de los TH realizados por estas indicaciones. Este trabajo fue presentado y aprobado por el comité de ética científica de la Facultad de Medicina de la Pontificia Universidad Católica de Chile.

\section{Análisis estadístico}

Se utilizó una estadística descriptiva y analítica. Se utilizaron medidas de tendencia central y de dispersión; para el cálculo y comparación de sobrevida se utilizaron curvas de Kaplan-Meier y test de $l o g$ rank respectivamente. En el análisis estadístico se utilizó el programa $\operatorname{SPSS}^{\circledR}$ v.20.

\section{Resultados}

\section{Indicaciones de $\mathrm{TH}$}

En el período estudiado se efectuaron $295 \mathrm{TH}$ en 261 pacientes; $34(11,5 \%)$ de ellos por alguna indicación inhabitual. En 7 casos el TH fue realizado en pacientes pediátricos $(20,5 \%)$ y 27 correspondieron a pacientes adultos $(79,5 \%)$. Las indicaciones de $\mathrm{TH}$ se describen en las Tablas 1 y 2 . Entre los pacientes con síndrome porto/hepatopulmonar, se trasplantó un enfermo con complicaciones graves de la cirrosis por virus $\mathrm{C}$ portador de una hemofilia $\mathrm{A}$ grave.

\section{Tabla 1. Indicaciones especiales de TH en pacientes} adultos

\begin{tabular}{|lc|}
\hline Indicación & n \\
\hline Síndrome porto/hepatopulmonar & 11 \\
Enfermedad poliquística & 9 \\
\hline Metástasis de tumor neuroendocrino & 3 \\
Enfermedad de Niemann-Pick & 2 \\
\hline Síndrome de Rendu-Osler-Weber & 1 \\
Metástasis hepáticas de GIST gástrico* & 1 \\
Total & 27 \\
\hline
\end{tabular}

*GIST $=$ Gastrointestinal stromal tumor.

Tabla 2. Indicaciones especiales de TH en pacientes pediátricos

\begin{tabular}{|ll|}
\hline Indicación & n \\
\hline Hepatoblastoma & 3 \\
\hline Déficit de Ornitin-Transcarbamilasa & 2 \\
\hline Síndrome de Alagille & 1 \\
Enfermedad de Niemann-Pick & 1 \\
Total & 7 \\
\hline
\end{tabular}


También se incluyó dentro de las indicaciones inhabituales, a un paciente con metástasis hepáticas irresecables de un tumor del estroma gastrointestinal (GIST) gástrico, resecado su tumor primario cinco años previo al trasplante. Su indicación de trasplante fue debida al masivo volumen hepático que estas metástasis presentaban, sin otras localizaciones secundarias extrahepáticas (Figura 1). Este paciente no presentaba insuficiencia hepática, como tampoco aquellos con enfermedad poliquística hepática (EPH), en quienes la indicación de su trasplante estuvo dada por la mala calidad de vida derivada de la masa y peso del hígado de los enfermos y su relación al volumen abdominal de cada paciente. En este grupo, el peso promedio del hígado explantado fue de $6.302 \mathrm{~g}$ con un rango que varió entre los 2.335 y los 15.000 g. En 4 casos de EPH se realizó trasplante combinado hepatorrenal por compromiso grave de la función renal. En otro paciente con indicación de trasplante combinado, no se realizó trasplante renal por inestabilidad hemodinámica persistente y coagulopatía en el intraoperatorio del trasplante de hígado, decisión tomada en conjunto con el equipo de trasplante renal.

En $5(14,7 \%)$ pacientes entre adultos y niños, la indicación del TH fue una enfermedad metabólica: 3 por enfermedad de Niemann-Pick y 2 por déficit de ornitintranscarbamilasa (enfermedad del ciclo de la urea), y en $7(20,6 \%)$ los pacientes tenían un tumor maligno primario o secundario del hígado.

\section{Características biodemográficas}

En el grupo pediátrico, la mediana de edad al momento del TH fue de 47 meses (rango: 6-192 meses); 5 (71,4\%) eran varones. En 4, el injerto se obtuvo de un donante vivo relacionado y en 3 de un donante fallecido. Previo al TH, tres niños con hepatoblastoma recibieron quimioterapia y en uno, además, se había practicado una hepatectomía derecha, con foco microscópico de tumor positivo en el borde quirúrgico.

De los 27 adultos, 16 (59,2\%) eran mujeres. La edad media al momento del TH de ellos era de 52 \pm 30 años.

Figura 1. A: Corte axial de $C T$ de abdomen en paciente con metástasis hepáticas de GIST gástrico. B: Corte coronal en mismo paciente. C: Corte axial en paciente con hígado con enfermedad poliquística. D: Corte coronal en mismo paciente.

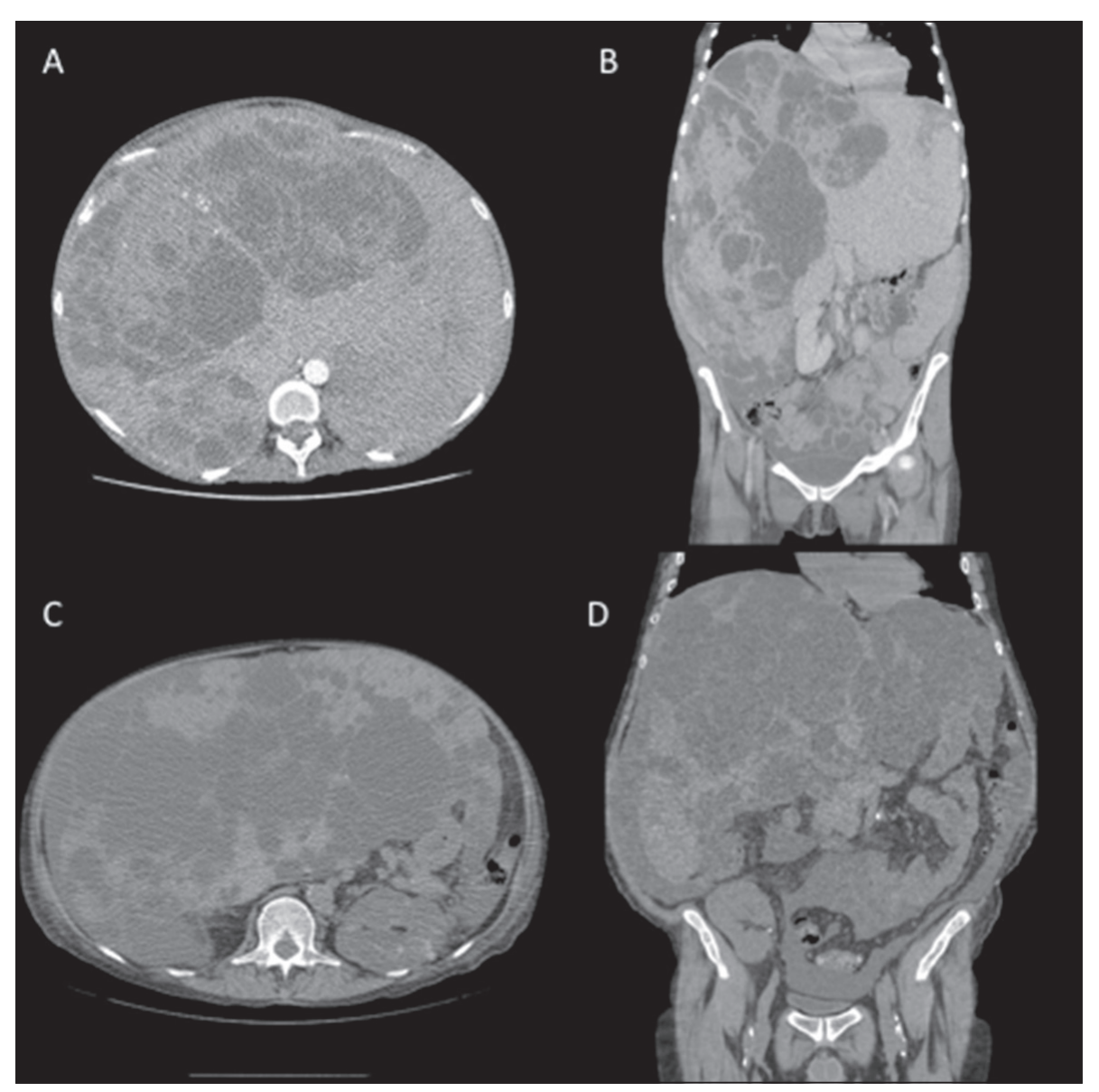




\section{Análisis de morbilidad y mortalidad}

La morbilidad observada en los 27 pacientes adultos se presenta en la Tabla 3. Destacan 4 $(14,8 \%)$ pacientes con trombosis de la anastomosis de la arteria hepática todos ellos portadores de una EPH; 2 de ellos requirieron un retrasplante, uno en 2 oportunidades que finalmente falleció.

Entre las complicaciones biliares, cabe destacar que otro paciente presentó una estenosis de la anastomosis más tardíamente (a los 7 meses post $\mathrm{TH}$ ), requiriendo tratamiento endoscópico.

Dos casos $(7,1 \%)$ presentaron rechazo agudo confirmado por histopatología, siendo tratados con bolos de corticoides y ajuste de inmunosupresión.

La morbilidad de los menores de 18 años se describe en la Tabla 4. Un paciente presentó un rechazo agudo que requirió de bolos de corticoides y ajuste de la inmunosupresión. En este grupo hubo 1 retrasplante en un paciente que presentó falla primaria del injerto (hígado de donante fallecido reducido) y una mortalidad tardía derivada de una complicación de la quimioterapia adyuvante a los 6 meses post $\mathrm{TH}$ en un paciente con un hepatoblastoma.

En total, 4 (12,5\%) pacientes necesitaron 5 retrasplantes, dos del grupo de enfermedad poliquística (uno de los cuales fue retrasplantado en 2 oportunidades), 1 del grupo de síndrome porto/hepatopulmonar que presentó una colangiopatía isquémica difusa a los 4 meses post TH y el paciente pediátrico descrito previamente.

La mortalidad a 90 días es de 2 pacientes $(5,9 \%)$, 1 paciente falleció en el intraoperatorio por un síndrome noradrenérgico refractario que era portador de metástasis hepáticas bilobares masivas de un tumor neuroendocrino (TNE) y el otro por un tromboembolismo pulmonar. Hubo, además, 2 fallecimientos posteriores a los 3 meses, de complicaciones graves. Al comparar las curvas de sobrevida, no se observaron diferencias entre los pacientes con indicación clásica de TH versus aquellos por indicaciones inhabituales (Figura 2).

El paciente con hemofilia grave trasplantado por síndrome porto/hepatopulmonar, vive sin dependencia de oxígeno, con el virus $\mathrm{C}$ erradicado definitivamente y sin manifestaciones clínicas de su hemofilia A, no requiriendo aportes de Factor VIII ${ }^{4}$. El paciente trasplantado por un hígado metastásico masivo por un GIST gastrointestinal de bajo grado está actualmente sin evidencia de recurrencia de enfermedad a 36 meses post $\mathrm{TH}$ con excelente calidad de $v i^{5}{ }^{5}$. Finalmente, los 3 pacientes con enfermedad de Niemann-Pick llevan una vida completamente normal con una inmunosupresión baja ( 2
Tabla 3. Morbilidad en pacientes adultos

\begin{tabular}{|c|c|c|}
\hline Tipo de morbilidad & $\mathbf{n}$ & Tratamiento \\
\hline $\begin{array}{l}\text { Vascular } \\
\text { - Trombosis arteria hepática }\end{array}$ & 4 & $\begin{array}{l}\text { Retrasplante (2), revascularización (1), } \\
\text { fallecido a los } 5 \text { meses (1) }\end{array}$ \\
\hline $\begin{array}{l}\text { Biliar } \\
\text { - Estenosis anastomosis } \\
\text { - Bilirragia }\end{array}$ & $\begin{array}{l}2 \\
2\end{array}$ & $\begin{array}{l}\text { Prótesis biliar (2), } 1 \text { detectada a los } 7 \text { meses } \\
\text { Tratamiento conservador (2) }\end{array}$ \\
\hline $\begin{array}{l}\text { Infecciosa } \\
\text { - Infección catéter central } \\
\text { - Colección abdominal } \\
\text { - Neumonía } \\
\text { - Infección urinaria }\end{array}$ & $\begin{array}{l}1 \\
1 \\
1 \\
1\end{array}$ & $\begin{array}{l}\text { Antibioticoterapia } \\
\text { Antibioticoterapia y drenaje percutáneo } \\
\text { Antibioticoterapia } \\
\text { Antibioticoterapia }\end{array}$ \\
\hline $\begin{array}{l}\text { Inmunológica } \\
\text { • Rechazo celular agudo }\end{array}$ & 2 & $\begin{array}{l}\text { Bolos esteroidales y readecuación de } \\
\text { esquema IS* }\end{array}$ \\
\hline $\begin{array}{l}\text { Otras } \\
\quad \text { - Hemoperitoneo } \\
\text { - Hematoma subcapsular }\end{array}$ & $\begin{array}{l}3 \\
1\end{array}$ & $\begin{array}{l}\text { Reexploración quirúrgica (3) } \\
\text { Drenaje abierto }\end{array}$ \\
\hline
\end{tabular}

* IS: Inmunosupresor.

Tabla 4. Morbilidad en pacientes pediátricos

\begin{tabular}{|lll|}
\hline Tipo de morbilidad & n & Tratamiento \\
Vasculares & 0 & $(-)$ \\
\hline $\begin{array}{l}\text { Biliares } \\
\text { - Biliperitoneo } \\
\text { - Biloma }\end{array}$ & 1 & $\begin{array}{l}\text { Reexploración } \\
\text { Infecciosas } \\
\text { - Shock séptico }\end{array}$ \\
$\begin{array}{l}\text { Otras } \\
\text { - Hemoperitoneo }\end{array}$ & 1 & Punción percutánea \\
\hline
\end{tabular}

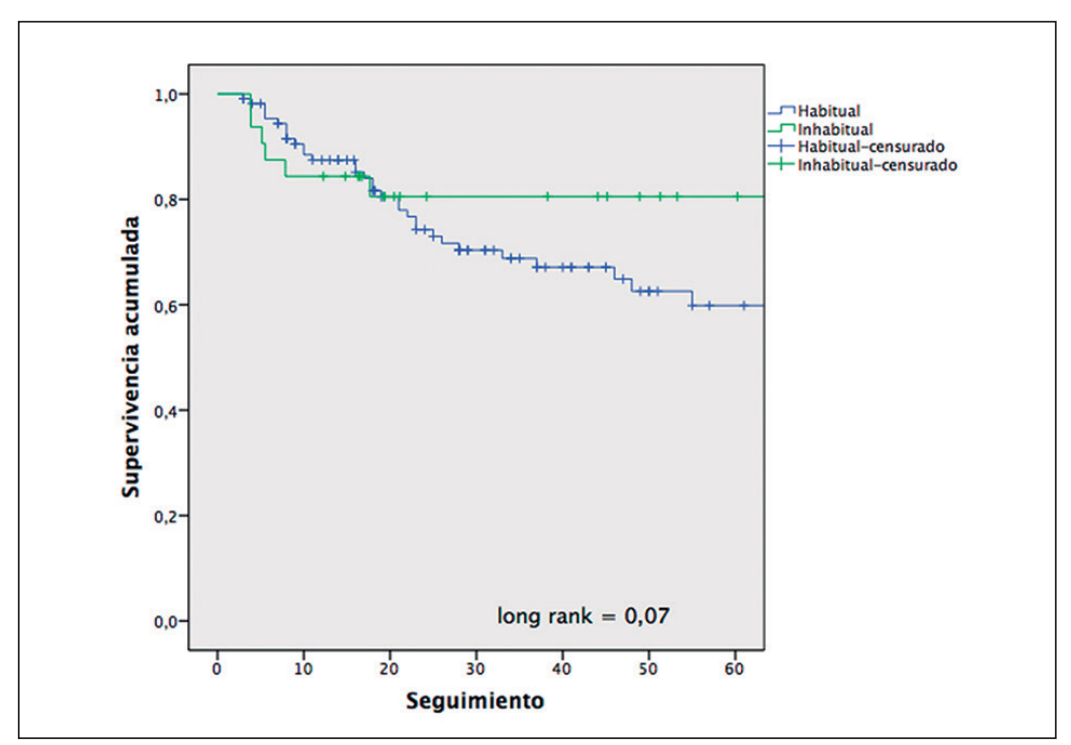

Figura 2. Comparación de sobrevida indicaciones especiales vs clásicas. 
pacientes en monoterapia con tacrolimus; 1 paciente en biterapia con ciclosporina y azatioprina en dosis mínimas).

\section{Discusión}

Esta serie es, a nuestro conocimiento, la primera en Chile que presenta resultados de indicaciones inhabituales del TH. Hace 10 años, un trabajo multicéntrico chileno mostró las indicaciones y resultados del $\mathrm{TH}^{6}$. Entre éstas, las más frecuentes en adultos eran: cirrosis de distintas etiologías, hepatitis autoinmune, virus $\mathrm{C}$, cirrosis biliar primaria y hepatitis fulminante; $y$, en niños, atresia de vías biliares y hepatitis fulminante ${ }^{6}$.

La principal indicación de TH de las enfermedades hepáticas crónicas cirróticas no colestásicas es la insuficiencia hepática crónica la cual se cuantifica a través de los scores de Child-Pugh ${ }^{7}$ y desde el año 2012 en nuestro país por el score de MELD $^{8}$. Entre las complicaciones más frecuentes de la cirrosis están aquellas secundarias a la hipertensión portal, como hemorragias digestivas no manejables con otras terapias, ascitis refractaria, hidrotórax hepático, peritonitis bacteriana del cirrótico a repetición y encefalopatía crónica. Una complicación poco frecuente, pero grave, es la insuficiencia respiratoria asociada a 2 condiciones pulmonares principales. Estas corresponden al síndrome hepatopulmonar (SHP) e hipertensión portopulmonar (HPP). El SHP se caracteriza por una anormal oxigenación arterial causada por dilataciones vasculares intrapulmonares en el contexto de enfermedad hepática avanzada, hipertensión portal o shunts portosistémicos congénitos $^{9}$. La oxigenación anormal se define por una elevada gradiente alvéolo-arterial $(\geq 15 \mathrm{mmHg}$ o $\geq 20 \mathrm{mmHg}$ si el paciente es mayor de 64 años) sin suplemento de oxígeno, en posición sentada o en reposo), en ausencia de otras enfermedades pulmonares que puedan alterar los test de función pulmonar9. Las dilataciones vasculares intrapulmonares se pueden detectar de manera óptima a través de un ecocardiograma transtorácico ${ }^{9}$. Su única terapia efectiva es el $\mathrm{TH}$, con recuperación completa de la función pulmonar a 1 año y una sobrevida a 5 años de $76 \%$, lo cual es similar a otras indicaciones electivas sin $\mathrm{SHP}^{9-11}$. Swanson et al. ${ }^{12}$, en una serie de 37 pacientes logró un $80 \%$ de resolución completa del SHP posterior al TH, con una mortalidad de $16 \%$ a los 3 meses. La HPP se diagnostica a través de criterios hemodinámicos obtenidos a través de mediciones por cateterismo en el corazón derecho por un catéter de Swan-Ganz ${ }^{9}$. El aumento de presión media de la arteria pulmonar debido a aumento en la resistencia vascular pulmonar en el contexto de una presión normal de enclavamiento es la piedra angular del diagnóstico de HPP ${ }^{9}$. Nuestro grupo de pacientes con SHP y/o HPP, presentaban todos una cirrosis hepática, pero la indicación del TH fue por la insuficiencia respiratoria y no por la insuficiencia hepática.

En relación a la enfermedad poliquística hepática (EPH), esta es una entidad infrecuente, de etiología genética autosómica dominante que puede estar asociada o no a las mutaciones de los genes PKD1 y $P K D 2$ descritos para la enfermedad autosómica dominante de la poliquistosis renal ${ }^{13,15}$. La EPH se caracteriza por la presencia de múltiples quistes de diverso tamaño en el parénquima hepático sin insuficiencia hepática, lo que lleva al crecimiento de la glándula en forma asintomática por muchos años lo que da cuenta de que la indicación en esta entidad sea el gran volumen hepático por síntomas compresivos debido a la hepatomegalia ocasionando dolor abdominal, dolor lumbar, disnea, saciedad precoz y desnutrición. El TH es considerado el único tratamiento curativo para la $\mathrm{EPH}$, el cual ha sido reservado para pacientes sintomáticos que no han respondido a tratamiento quirúrgico alternativos al trasplante (como fenestración laparoscópica, resecciones hepáticas e intervenciones percutáneas). El TH conlleva una mejoría sustancial en la calidad de vida de estos pacientes, sin embargo, sus dificultades técnicas debido al gran tamaño del hígado, pueden acompañarse de una alta morbimortalidad asociada la intervención. En esta serie tuvimos mayor incidencia de trombosis de la arteria hepática asociada a los $\mathrm{TH}$ por esta indicación con 2 retrasplantes y una mortalidad en 10 pacientes. Sin embargo, estos resultados son similares a estudios previamente publicados ${ }^{11-13}$.

La enfermedad de Niemann-Pick es un síndrome infrecuente causado por la acumulación intracelular anormal de lípidos (principalmente esfingomielina y colesterol) en órganos como el bazo y el hígado ${ }^{15}$. Puede comenzar en la infancia evolucionando con falla orgánica rápidamente progresiva ocasionando la muerte o de manera insidiosa e instalación más tardía $^{15}$. Se ha clasificado en 6 variantes de A a F, siendo las más frecuentes: $\mathrm{A}, \mathrm{B}$ y $\mathrm{C}^{15}$. La enfermedad de Niemann-Pick tipo B es caracterizada por hepatoesplenomegalia, la cual puede llevar a una cirrosis hepática secundaria. Existen pocos artícu$\operatorname{los}^{15,16}$ de TH por esta patología, generalmente en pacientes pediátricos, cuya indicación es la progresión a falla hepática terminal. En esta serie presentamos 3 pacientes con $\mathrm{TH}$ por esta etiología con 
resultados favorables, actualmente con dosis bajas de inmunosupresores y una óptima calidad de vida.

Los TNE tienen una baja incidencia $(<5$ por 100.000 habitantes) y de acuerdo a la Organización Mundial de la Salud (OMS) se clasifican de acuerdo a su índice mitótico y el Ki-67 en bajo, intermedio y alto grado ${ }^{17}$. El $\mathrm{TH}$ debe ser considerado una alternativa terapéutica en pacientes con TNE metastásicos al hígado irresecables con las siguientes características: bien diferenciados (grado bajo o intermedio), con un $\mathrm{Ki}-67<10 \%$, con hasta un $75 \%$ de compromiso hepático, sin enfermedad extrahepática, edad $<55$ años, tumor primario tratado pre TH y enfermedad estable en los últimos 6 meses $^{17-19}$. Mazzaferro $^{19}$, en 2016, reportó la serie más grande de pacientes con TH por metástasis de TNE $(n=24)$ con una sobrevida a 5 años similar a lo descrito para otros TH $(89 \%)$.

Los TH de otras etiologías mencionadas en esta serie de pacientes (Rendu-Osler-Weber, Alagille y déficit de ornitin-transcarbamilasa) son anecdóticos y principalmente se publican como casos clínicos o serie de $\operatorname{casos}^{2,20,21}$. Estas indicaciones, tal como se ha hecho énfasis deben ser analizadas de manera conjunta por equipos multidisciplinarios, ya que si bien muchos de estos pacientes no tienen insuficiencia hepática, sí presentarán una mejoría fundamental en su calidad de vida posterior al TH.

El hepatoblastoma es el tumor más frecuente en edad pediátrica, siendo diagnosticado generalmente antes de los 5 años de edad ${ }^{22}$. El tratamiento consiste en una combinación de quimioterapia más cirugía (resección hepática o TH). En esta serie, los 3 pacientes recibieron quimioterapia previa y en uno de ellos, además, se realizó una hepatectomía derecha.
La resección completa es el punto crítico para la terapia de estos pacientes, sin embargo, casi el 60\% de los tumores es irresecable al momento del diagnóstico, estableciendo al TH como la única alternativa de curación ${ }^{22,23}$. La incidencia de este tumor se ha incrementado lo que, asociado a la experiencia adquirida en $\mathrm{TH}$ con donantes vivos, da cuenta del mayor número de TH por esta indicación. En los 3 casos de esta serie, se realizó TH con donante vivo. La sobrevida publicada, a partir de 300 casos hasta el año 2016, se describe en un $76 \%$ a 5 años ${ }^{2,22,23}$.

En conclusión, el TH parece ser una opción factible y se encuentra asociada a una mejor calidad de vida en pacientes con esta miscelánea de indicaciones. En enfermedades hepáticas no cirróticas el TH trata las funciones hepáticas alteradas y previene el riesgo de malignidad. La selección de los pacientes, el momento del $\mathrm{TH}$, los cuidados pre y postoperatorios son claves para disminuir la morbimortalidad. De acuerdo a nuestros resultados, las indicaciones inhabituales o infrecuentes de TH, deben ser consideradas en la estrategia terapéutica de cada una de ellas.

\section{Responsabilidades éticas}

Protección de personas y animales. Los autores declaran que para esta investigación no se han realizado experimentos en seres humanos ni en animales.

Confidencialidad de los datos. Los autores declaran que en este artículo no aparecen datos de pacientes.

Conflictos de interés: no hay.

\section{Bibliografía}

1. Sociedad Chilena de Trasplante. Guía Clínica Trasplante Hepático. 2009:315412 .

2. Akdur A, Kirnap M, Soy EHA, Ozcay F, Moray G, Arslan G, et al. Unusual indications for a liver transplant: A singlecenter experience. Exp Clin Transplant. 2017;15:128-32.

3. Martínez J, Jarufe N, Crovari F, Álvarez S, Arrese M, Pérez RM, et al. Procura de hígado para trasplante hepático ortotópico. Comparación de dos técnicas. Rev Chil Cir. 2005;57:26-32.

4. Benítez C, Zúñiga $P$, Kramer F, Valladares X, Rojas P, Pimentel E, et al. El trasplante hepático es un procedimiento plausible, efectivo y seguro en pacientes con hemofilia. Rev Med Chile 2019;147:37883.

5. Martínez J, Besa S, Arab JP, Quintana JC, Regonesi C, Huete A, et al. Gastrointestinal neuroendocrine tumor with unresectable liver metastases: an example of multimodal therapeutic approach. Ann Hepatol. 2015;14:752-5.

6. Hepp J, Zapata R, Buckel E, Martínez J, Uribe M, Díaz JC, et al. Trasplante hepático en Chile: Aspectos generales, indicaciones y contraindicaciones (Documento de consenso). Rev Med Chile 2008;136:793-804.

7. Pugh RNH, Murray-Lyon IM, Dawson JL,
Pietroni MC, Williams R. Transection of the oesophagus for bleeding oesophageal varices. Br J Surg. 60:646-9.

8. Malinchoc M, Kamath PS, Gordon FD, Peine CJ, Rank J, Ter Borg PCJ. A model to predict poor survival in patients undergoing transjugular intrahepatic portosystemic shunts. Hepatology 2000;31:864-71.

9. Krowka MJ, Fallon MB, Kawut SM, Fuhrmann V, Heimbach JK, Ramsay MA, et al. International Liver Transplant Society Practice Guidelines: Diagnosis and Management of Hepatopulmonary Syndrome and Portopulmonary Hypertension. Transplantation 2016;100:1440-52. 
10. Cosarderelioglu C, Cosar AM, Gurakar M, Dagher NN, Gurakar A. Hepatopulmonary Syndrome and Liver Transplantation: A Recent Review of the Literature. J Clin Transl Hepatol. 2016;4:47-53.

11. Liberal R, Grant CR, Baptista R, Macedo G. Porto-pulmonary hypertension: A comprehensive review. Clin Res Hepatol Gastroenterol. 2015;39:157-67.

12. Swanson KL, Wiesner RH, Krowka MJ Natural history of hepatopulmonary syndrome: Impact of liver transplantation. Hepatology 2005;41:1122-9.

13. Baber JT, Hiatt JR, Busuttil RW, Agopian VG. A 20-year experience with liver transplantation for polycystic liver disease: Does previous palliative surgical intervention affect outcomes? J Am Coll Surg. 2014;219:695-703.

14. Zamora-Valdés D, Contreras AG, Mercado MA. Liver transplantation for polycystic liver disease. J Am Coll Surg. 2014;219:1192.
15. Coelho GR, Praciano AM, Rodrigues JPC, Viana CF, Brandao KP, Valenca JT, et al. Liver Transplantation in Patients With Niemann-Pick Disease - SingleCenter Experience. Transplant Proc. 2015;47:2929-31.

16. Mendes MS, Portela FX, Reis RC, Castro JD, García JH, Holanda MA. Liver transplantation in a patient with NiemannPick disease and pulmonary involvement. J Bras Pneumol. 2012;38:269-71.

17. Öberg K, Castellano D. Current knowledge on diagnosis and staging of neuroendocrine tumors. Cancer Metastasis Rev. 2011;30(Suppl. 1):3-7.

18. Mazzaferro V, Pulvirenti A, Coppa J. Neuroendocrine tumors metastatic to the liver: How to select patients for liver transplantation? J Hepatol. 2007;47:460-6.

19. Mazzaferro V, Sposito C, Coppa J, Miceli $\mathrm{R}$, Bhoori S, Bongini M, et al. The LongTerm Benefit of Liver Transplantation for Hepatic Metastases From Neuroendocrine
Tumors. Tumors. Am J Transplant. 2016;16:2892-902.

20. Scelzo C, Greco S, Bonanni L, Di Cocco P, D’Angelo M, Laurenzi C, et al. The Role of Liver Transplantation in the Treatment of Hereditary Hemorrhagic Telangiectasia: A Short Literature Review. Transplant Proc. 2007;39:2045-7.

21. Yu L, Rayhill SC, Hsu EK, Landis CS. Liver Transplantation for Urea Cycle Disorders: Analysis of the United Network for Organ Sharing Database. Transplant Proc. 2015;47:2413-8.

22. Trobaugh-Lotrario AD, Meyers RL, Tiao GM, Feusner JH. Pediatric liver transplantation for hepatoblastoma. Transl Gastroenterol Hepatol. 2016;1:44.

23. Okur MH, Yankol Y, Cimşit B, Mecit N, Ertugrul G, Kanmaz T, et al. Liver Transplant in Children with Hepatoblastoma. 2019;17:6447. DOI: 10.6002/ect.2016.0110. Epub 2017 Apr 14. 